\title{
On Commutativity of Semiprime Rings with Multiplicative (generalized)-derivations
}

\author{
Deepak Kumar ${ }^{1} \&$ Gurninder S. Sandhu ${ }^{1}$ \\ ${ }^{1}$ Department of Mathematics, Punjabi University, Patiala, Punjab, India \\ Correspondence: Gurninder S. Sandhu, Department of Mathematics, Punjabi University, Patiala. E-mail: sandhugurnin- \\ der@gmail.com
}

Received: December 5, 2016 Accepted: January 18, 2017 Online Published: March 5, 2017

doi:10.5539/jmr.v9n2p9 URL: https://doi.org/10.5539/jmr.v9n2p9

\begin{abstract}
The aim of this paper is to explore the commutativity of semiprime rings admitting multiplicative (generalized)-derivations and satisfy certain hypotheses on appropriate subsets.
\end{abstract}

Keywords: semiprime ring, ideals, derivation, multiplicative (generalized)-derivation.

\section{Introduction}

Throughout this paper $R$ denotes an associative ring with center $Z(R)$. Recall, a ring $R$ is said to be prime ring if for any $a, b \in R, a R b=(0)$ implies either $a=0$ or $b=0$ and is semiprime ring if $a R a=(0)$ implies $a=0$. For any $x, y \in R$, we shall denote the commutator and anti-commutator by the symbols $[x, y]=x y-y x$ and $(x \circ y)=x y+y x$ respectively. We shall frequently use the basic commutator and anti-commutator identities : $[x y, z]=x[y, z]+[x, z] y,[x, y z]=y[x, z]+[x, y] z$ and $(x \circ y z)=(x \circ y) z-y[x, z]=y(x \circ z)+[x . y] z,(x y \circ z)=x(y \circ z)-[x, z] y=(x \circ z) y+x[y, z]$. An additive map $f: R \rightarrow R$ is called a derivation of $R$ if $f(x y)=f(x) y+x f(y)$ holds for all $x, y \in R$. Let $F: R \rightarrow R$ be a map together with another map $f: R \rightarrow R$ so that $F(x y)=F(x) y+x f(y)$ for all $x, y \in R$. If $F$ is additive and $f$ a derivation of $R$, then $F$ is called generalized derivation of $R$ and if $f=0$, then $F$ is called left multiplier of $R$. The notion of generalized derivation was introduced by Brešar (Brešar, 1991). In (Havala, 1998), author gave an algebraic study of these mappings in prime rings. Obviously, every derivation is a generalized derivation. In this way generalized derivation covers both concepts of derivation and left multiplier of $R$. Let $K$ be a nonempty subset of $R$, a map $f: K \rightarrow R$ is said to be centralizing on $K$, if $[f(x), x] \in Z(R)$ for all $x \in K$. In particular, if $[f(x), x]=0$ for all $x \in K$, then $f$ is called commuting on $K$.

In the literature, a number of authors have discussed the commutativity of prime rings and semiprime rings admitting derivations and generalized derivations satisfying certain algebraic identities, see (Ali, Kumar \& Miyan, 2011), (Ali, Dhara \& Fos̆ner, 2011), (Andima \& Pajoohesh, 2010), (Ashraf et al, 2007, 2001), (Daif \& Bell, 1992), (Dhara \& Pattanayak, 2011), (Hongan, 1997), where further references can be found.

Let us swing to the foundation examination of multiplicative (generalized)-derivations of associative rings. Inspired by the work of Martindale III (Martindale, 1969), Daif (Daif, 1991) introduced the concept of multiplicative derivations. Accordingly, a map $f: R \rightarrow R$ is called multiplicative derivation of $\mathrm{R}$ if $f(x y)=f(x) y+x f(y)$ holds for all $x, y \in R$. Of course, these maps are not necessarily additive. Goldmann and Sěmrl (Goldmann \& Sěmrl, 1996) presented complete description of these maps. Further, Daif and Tammam-El-Sayiad (Daif \& Tammam-El-Sayiad, 1997) extended the notion of multiplicative derivation to multiplicative generalized derivation as follows: A map $F: R \rightarrow R$ is called multiplicative generalized derivation of $\mathrm{R}$ if $F(x y)=F(x) y+x f(y)$ holds for all $x, y \in R$, where $f$ is a derivation of R. Recently, Dhara and Ali (Dhara \& Ali, 2013) made a slight generalization in above definition of multiplicative generalized derivation by relaxing the conditions on $f$. A map $F: R \rightarrow R$ (not necessarily additive) is said to be a multiplicative (generalized)derivation if $F(x y)=F(x) y+x f(y)$ holds for all $x, y \in R$, where $f$ can be any map (not necessarily additive nor a derivation). For convenience we denote it by a pair $(F, f)$. In the previous couple of years many outcomes has been gotten in prime and semi-prime rings involving multiplicative (generalized)-derivations, see (Ali et al, 2015), (Ali et al, 2014), (Dhara \& Ali, 2013), (Dhara et al, 2014) and (Khan, 2016). As multiplicative (generalized)-derivation is an extended notion of generalized derivation, so it is noteworthy to demonstrate the consequences of generalized derivations for multiplicative (generalized)-derivations.

The main objective of this paper is to take care of the issue raised by author in (Khan, 2016) and investigate the commutativity of $R$. Precisely, we concentrate on the following central-valued conditions: $f(x) F(y) \pm y x \in Z(R)$, $f(x) F(y) \pm x y \in Z(R), f(x) F(y) \pm(x \circ y) \in Z(R), f(x) F(y) \pm[x, y] \in Z(R), F(x y) \pm F(x) F(y) \in Z(R), F[x, y] \pm(x \circ y) \in Z(R)$, $F(x \circ y) \pm[x, y] \in Z(R), F[x, y] \pm x y \in Z(R), F(x \circ y) \pm x y \in Z(R), F[x, y] \pm f(x) \circ y \in Z(R), F(x \circ y) \pm[f(x), y] \in Z(R)$ where $x$ and $y$ are from an appropriate subset of $R$. 


\section{Main Results}

Theorem 1. Let $R$ be a semiprime ring and I a nonzero ideal of $R$. Suppose that $(F, f)$ is a multiplicative (generalized)derivation of $R$. If $f(x) F(y) \pm y x \in Z(R)$ for all $x, y \in I$, then $f$ is commuting on I and I is commutative.

Proof. We consider

$$
f(x) F(y) \pm y x \in Z(R) \text { for all } x, y \in I .
$$

Replace $y$ by $y z$ in (1) to get $(f(x) F(y) \pm y x) z+f(x) y f(z) \pm y[z, x] \in Z(R)$ for all $x, y, z \in I$. On commuting with $z$ we obtain

$$
[f(x) y f(z), z] \pm[y[z, x], z]=0 \text { for all } x, y, z \in I .
$$

In particular, putting $x=z$ to obtain

$$
[f(z) y f(z), z]=0 \text { for all } y, z \in I
$$

Which implies that

$$
f(z) y f(z) z=z f(z) y f(z) \text { for all } x, y, z \in I
$$

Substituting $y f(z) w$ for $\mathrm{y}$ in (4), we have

$$
f(z) y f(z) w f(z) z=z f(z) y f(z) w f(z) \text { for all } x, y, z, w \in I .
$$

Using (4) in (5), we obtain $f(z) y z f(z) w f(z)=f(z) y f(z) z w f(z)$ for all $x, y, z, w \in I$. That is $x f(z) y[f(z), z] w f(z)=0$ for all $x, y, z, w \in I$. It implies that $x[f(z), z] y[f(z), z] w[f(z), z]=0$ for all $x, y, z, w \in I$. Therefore, $(I[f(z), z])^{3}=(0)$ for all $z \in I$. But R has no nonzero nilpotent ideal, we conclude that $I[f(z), z]=(0)$ for all $z \in I$. Thus, $[f(z), z]=0$ for all $z \in I$ (See, (Herstein, 1976)).

Now, Replace $y$ by $y z$ in (2) and we get

$$
[f(x) y z f(z), z] \pm[y z[z, x], z]=0 \text { for all } x, y, z \in I .
$$

Right multiply (2) by $z$ and subtract (6) from it, we obtain $[f(x) y[f(z), z], z] \pm[y[[z, x], z], z]=0$ for all $x, y, z \in I$. Using the fact that $I[f(z), z]=(0)$ for all $z \in I$, we get

$$
[y[[z, x], z], z]=0 \text { for all } x, y, z \in I .
$$

Replace $y$ by $x y$ in (7), we obtain

$$
x[y[[z, x], z], z]+[x, z] y[[z, x], z]=0 \text { for all } x, y, z \in I .
$$

Using (7), it reduces to

$$
[x, z] y[[z, x], z]=0 \text { for all } x, y, z \in I .
$$

Replace $y$ by $z y$ in (9), we get

$$
[x, z] z y[[x, z], z]=0 \text { for all } x, y, z \in I
$$

Left multiply (9) by $z$ and subtract from (10), we get $[[x, z], z] y[[x, z], z]=0$ for all $x, y, z \in I$. That is $[[x, z], z] I[[x, z], z]=$ (0) for all $x, z \in I$. Semiprimeness of I yields that

$$
[[x, z], z]=0 \text { for all } x, z \in I .
$$

Linearizing (11) with respect to $\mathrm{z}$ and using (11), we have

$$
[[x, z], t]+[[x, t], z]=0 \text { for all } x, t, z \in I .
$$

Replace $z$ by $z t$ in (12), we get $z[[x, t], t]+[z, t][x, t]+([[x, z], t]+[[x, t], z]) t+z[[x, t], t]=0$ for all $x, t, z \in I$. Using (11) and (12), we obtain

$$
[z, t][x, t]=0 \text { for all } x, t, z \in I .
$$

Replace $x$ by $x y$ in (13) to get $[z, t] x[y, t]+[z, t][x, t] y=0$ for all $x, y, t, z \in I$. Using (13), we obtain $[z, t] x[y, t]=0$ for all $x, y, t, z \in I$. In particular, $[y, t] I[y, t]=(0)$ for all $y, t \in I$. It implies that $[y, t]=0$ for all $y, t \in I$. Hence, $[I, I]=(0)$ as desired.

Theorem 2. Let $R$ be a semiprime ring and I a nonzero ideal of $R$. Suppose that $(F, f)$ is a multiplicative (generalized)derivation of $R$. If $f(x) F(y) \pm x y \in Z(R)$ for all $x, y \in I$, then $f$ is commuting on $I$. 
Proof. We consider

$$
f(x) F(y) \pm x y \in Z(R) \text { for all } x, y \in I .
$$

Replace $y$ by $y z$ in (14), we get

$$
(f(x) F(y) \pm x y) z+f(x) y f(z) \in Z(R) \text { for all } x, y, z \in I .
$$

On commuting with $z$ in (15), we obtain $[f(x) y f(z), z]=0$ for all $x, y, z \in I$. In particular, put $x=z$, we get $[f(z) y f(z), z]$ for all $y, z \in I$. It coincides with (3), hence Theorem 1. insures the conclusion.

Theorem 3. Let $R$ be a semiprime ring and I a nonzero ideal of $R$. Suppose that $(F, f)$ is a multiplicative (generalized)derivation of $R$. If $f(x) F(y) \pm(x \circ y) \in Z(R)$ for all $x, y \in I$, then $f$ is commuting on I and I is commutative.

Proof. We consider

$$
f(x) F(y) \pm(x \circ y) \in Z(R) \text { for all } x, y \in I
$$

Replace $y$ by $y z$ in (16) to obtain $(f(x) F(y) \pm(x \circ y)) z+f(x) y f(z) \mp y[x, z] \in Z(R)$ for all $x, y, z \in I$. On commuting both sides by $z$, we get $[f(x) y f(z), z] \mp[y[z, x], z]=0$ for all $x, y, z \in I$. It coincides with (2), hence Theorem 1 . insure the conclusions.

Theorem 4. Let $R$ be a semiprime ring and I a nonzero ideal of $R$. Suppose that $(F, f)$ is a multiplicative (generalized)derivation of $R$. If $f(x) F(y) \pm[x, y] \in Z(R)$ for all $x, y \in I$, then $f$ is commuting on I and I is commutative.

Proof. We consider

$$
f(x) F(y) \pm[x, y] \in Z(R) \text { for all } x, y \in I
$$

Replace $y$ by $y z$ in (17) to obtain $(f(x) F(y) \pm[x, y]) z+f(x) y f(z) \pm y[x, z] \in Z(R)$ for all $x, y, z \in I$. On commuting both sides by $z$, we have

$$
[f(x) y f(z), z] \pm[y[x, z], z]=0 \text { for all } x, y, z \in I
$$

Substituting $x=z$ and we get $[f(z) y f(z), z]=0$ this is same as (3) so by theorem 1 , we obtain $[f(z), z]=0$ for all $z \in I$. Replace $y$ by $y z$ in (18), we get

$$
[f(x) y z f(z), z] \pm[y z[x, z], z]=0 \text { for all } x, y, z \in I
$$

Right multiply (18) by $z$ and subtract (19) from it and we get $[f(x) y[f(z), z], z] \pm[y[[x, z], z], z]=0$ for all $x, y, z \in I$. Using the fact that $I[f(z), z]=0$ for all $z \in I$, we obtain $[y[[x, z], z], z]=0$ for all $x, y, z \in I$. It coincides with (7), hence Theorem 1. insures the conclusion.

Corollary 5. Let $R$ be a semiprime ring. If $(F, f)$ is a multiplicative (generalized) -derivation of $R$ such that any one of the following

$$
\begin{aligned}
& \text { i. } f(x) F(y) \pm[x, y] \in Z(R) \\
& \text { ii. } f(x) F(y) \pm(x \circ y) \in Z(R) \\
& \text { iii. } f(x) F(y) \pm y x \in Z(R)
\end{aligned}
$$

holds for all $x, y \in R$, then $R$ is commutative.

Theorem 6. Let $R$ be a semiprime ring and I a nonzero left ideal of $R$. Suppose that $(F, f)$ is a multiplicative (generalized)derivation of $R$. If $F(x y) \pm F(x) F(y) \in Z(R)$ holds for all $x, y \in I$, then $I[f(z), z]=(0)$ for all $z \in I$.

Proof. We consider

$$
F(x y) \pm F(x) F(y) \in Z(R) \text { for all } x, y, z \in I .
$$

Replace $y$ by $y z$ in (20), we get $(F(x y) \pm F(x) F(y)) z+x y f(z) \pm F(x) y f(z) \in Z(R)$ for all $x, y, z \in I$. On commuting with $z$ and using (20), we obtain

$$
[x y f(z), z] \pm[F(x) y f(z), z]=0 \text { for all } x, y, z \in I .
$$

Replace $x$ by $x z$ in (21) to get

$$
[x z y f(z), z] \pm[F(x) z y f(z), z] \pm[x f(z) y f(z), z]=0 \text { for all } x, y, z \in I .
$$

Replace $y$ by $z y$ in (21) and subtract it from (22), we have

$$
[x f(z) y f(z), z]=0 \text { for all } x, y, z \in I \text {. }
$$


Substitute $f(z) x$ for $x$ in (23), we get $f(z)[x f(z) y f(z), z]+[f(z), z] x f(z) y f(z)=0$ for all $x, y, z \in I$. Relation (23) reduce it to

$$
[f(z), z] x f(z) y f(z)=0 \text { for all } x, y, z \in I .
$$

Replace $x$ by $x z$ in (24) and we get

$$
[f(z), z] x z f(z) y f(z)=0 \text { for all } x, y, z \in I \text {. }
$$

Replace $y$ by $y z$ in (24), we have

$$
[f(z), z] x f(z) z y f(z)=0 \text { for all } x, y, z \in I
$$

Subtract (25) from (26)to obtain $[f(z), z] x[f(z), z] y f(z)=0$ for all $x, y, z \in I$. It implies that $(I[f(z), z])^{3}=(0)$ for all $z \in I$. Hence, we conclude that $I[f(z), z]=(0)$ for all $z \in I$.

Corollary 7. Let $R$ be a semiprime ring and $(F, f)$ a multiplicative (generalized)-derivation of $R$. If $F(x y) \pm F(x) F(y) \in$ $Z(R)$ holds for all $x, y \in R$, then $f$ is a commuting map.

Theorem 8. Let $R$ be a semiprime ring and I a nonzero left ideal of $R$. Suppose that $(F, f)$ is a multiplicative (generalized)derivation of $R$. If $F[x, y] \pm(x \circ y) \in Z(R)$ for all $x, y \in I$, then $I[x, f(x)]=(0)$ or $I[x, f(Z(R))]=(0)$ for all $x \in I$.

Proof. We consider

$$
F[x, y] \pm(x \circ y) \in Z(R) \text { for all } x, y \in I
$$

If $Z(R)=(0)$ then

$$
F[x, y] \pm(x \circ y)=0 \text { for all } x, y \in I .
$$

Replace $y$ by $y x$ in (28) and we get $(F[x, y] \pm(x \circ y)) x+[x, y] f(x)=0$ for all $x, y \in I$. It reduces to

$$
[x, y] f(x)=0 \text { for all } x, y \in I
$$

Replace $y$ by $f(x) y$ in (29), we have $f(x)[x, y] f(x)+[x, f(x)] y f(x)=0$ for all $x, y \in I$. Using (29), we obtain

$$
[x, f(x)] y f(x)=0 \text { for all } x, y \in I .
$$

Replace $y$ by $y x$ in (30) and we get

$$
[x, f(x)] y x f(x)=0 \text { for all } x, y \in I .
$$

Right multiply (30) by $x$ and subtract from (31), to obtain $[x, f(x)] y[x, f(x)]=0$ for all $x, y \in I$. Since I is a left ideal of $\mathrm{R}$, so we have $y[x, f(x)] R y[x, f(x)]=(0)$ for all $x, y \in I$. Semiprimeness of R yields that $y[x, f(x)]=0$ for all $x, y \in I$. Hence, we conclude that $I[x, f(x)]=(0)$ for all $x \in I$.

If $Z(R) \neq(0)$ then there exist $0 \neq t \in Z(R)$. Replace $y$ by $y t$ in (27), we get $(F[x, y] \pm(x \circ y)) t+[x, y] f(t) \in Z(R)$ for all $x, y \in I$. Using (27), we get $[x, y] f(t) \in Z(R)$ for all $x, y \in I$. On commuting with $r \in R$, we have

$$
[[x, y] f(t), r]=0 \text { for all } x, y \in I \text { and } r \in R \text {. }
$$

Replace $x$ by $y x$ in (32), we get $[y[x, y] f(t), r]=y[[x, y] f(t), r]+[y, r][x, y] f(t)=0$ for all $x, y \in I$ and $r \in R$. Using (32), we obtain

$$
[y, r][x, y] f(t)=0 \text { for all } x, y \in I \text { and } r \in R \text {. }
$$

Replace $r$ by $p r$ in (33) where $p \in R$, we get $p[y, r][x, y] f(t)+[y, p] r[x, y] f(t)=0$ for all $x, y \in I$ and $r, p \in R$. Using (33), we get $[y, p] r[x, y] f(t)=0$ for all $x, y \in I$ and $r, p \in R$. Substitute $f(t) r$ for $r$ and in particular, we get $[x, y] f(t) R[x, y] f(t)=(0)$ for all $x, y \in I$. Semiprimeness of $\mathrm{R}$ implies that

$$
[x, y] f(t)=0 \text { for all } x, y \in I .
$$

Replace $y$ by $f(t) y$ in (34), we get $f(t)[x, y] f(t)+[x, f(t)] y f(t)=0$ for all $x, y \in I$. Equation (34) forces that $[x, f(t)] y f(t)=$ 0 for all $x, y \in I$. It implies $[x, f(t)] y[x, f(t)]=0$ for all $x, y \in I$. Since I is a left ideal of R so we have $y[x, f(t)] R y[x, f(t)]=$ (0) for all $x, y \in I$. Semiprimeness of R yields that $y[x, f(t)]=0$ for all $x, y \in I$ and $t \in Z(R)$. Hence, we conclude that $I[x, f(Z(R))]=(0)$ for all $x \in I$. 
Theorem 9. Let $R$ be a semiprime ring and I a nonzero left ideal of $R$. Suppose that $(F, f)$ is a multiplicative (generalized)derivation of $R$. If $F(x \circ y) \pm[x, y] \in Z(R)$ for all $x, y \in I$, then $I[x, f(x)]=(0)$ or $I[x, f(Z(R))]=(0)$ for all $x \in I$.

Proof. We consider

$$
F(x \circ y) \pm[x, y] \in Z(R) \text { for all } x, y \in I
$$

If $Z(R)=(0)$ then

$$
F(x \circ y) \pm[x, y]=0 \text { for all } x, y \in I .
$$

Replace $y$ by $y x$ in (36), we get $(F(x \circ y) \pm[x, y]) x+(x \circ y) f(x)=0$ for all $x, y \in I$. Using (36) to obtain

$$
(x \circ y) f(x)=0 \text { for all } x, y \in I
$$

Replace $y$ by $f(x) y$ in (37) and we get $f(x)(x \circ y) f(x)+[x, f(x)] y f(x)=0$ for all $x, y \in I$. Relation (37) implies that

$$
[x, f(x)] y f(x)=0 \text { for all } x, y \in I .
$$

Replace $y$ by $y x$ in (38), we obtain

$$
[x, f(x)] y x f(x)=0 \text { for all } x, y \in I .
$$

Right multiply (38) by $x$ and subtract from (39), we get $[x, f(x)] y[x, f(x)]=0$ for all $x, y \in I$. Since I is a left ideal of $\mathrm{R}$, so we have $y[x, f(x)] R y[x, f(x)]=(0)$ for all $x, y \in I$. Semiprimeness of R yields that $y[x, f(x)]=0$ for all $x, y \in I$. Hence, we conclude that $I[x, f(x)]=(0)$ for all $x \in I$.

If $Z(R) \neq(0)$ then there exist $0 \neq t \in Z(R)$. Replace $y$ by $y t$ in $(27)$ to get $(F[x, y] \pm(x \circ y)) t+(x \circ y) f(t) \in Z(R)$ for all $x, y \in I$. Using (27), we left with $(x \circ y) f(t) \in Z(R)$ for all $x, y \in I$. On commuting with $r \in R$, we obtain

$$
[(x \circ y) f(t), r]=0 \text { for all } x, y \in I \text { and } r \in R \text {. }
$$

Replace $y$ by $x y$ in (40), we get $x[(x \circ y) f(t), r]+[x, r](x \circ y) f(t)=0$ for all $x, y \in I$ and $r \in R$. Equation (40) reduce it to

$$
[x, r](x \circ y) f(t)=0 \text { for all } x, y \in I \text { and } r \in R .
$$

Replace $y$ by $p y$ in (41) where $p \in R$, we have $[x, r] p(x \circ y) f(t)+[x, r][x, p] y f(t)=0$ for all $x, y \in I$ and $r, p \in R$. Using the fact that $(x \circ y) f(t) \in Z(R)$ for all $x, y \in I$, we get $[x, r](x \circ y) f(t) p+[x, r][x, p] y f(t)=0$ for all $x, y \in I$ and $r, p \in R$. Using (41) to obtain

$$
[x, r][x, p] y f(t)=0 \text { for all } x, y \in I \text { and } r, p \in R \text {. }
$$

Replacing $r$ by $s r$ where $s \in R$ in (42) and we have $s[x, r][x, p] y f(t)+[x, s] r[x, p] y f(t)=0$ for all $x, y \in I$ and $p, r, s \in R$. Using (42) to obtain

$$
[x, s] r[x, p] y f(t)=0 \text { for all } x, y \in I \text { and } p, r, s \in R .
$$

Replace $y$ by $y x$ in (43), we get

$$
[x, s] r[x, p] y x f(t)=0 \text { for all } x, y \in I \text { and } p, r, s \in R \text {. }
$$

Right multiply (43) by $x$ and subtract from (44) to get $[x, s] r[x, p] y[x, f(t)]=0$ for all $x, y \in I$ and $p, r, s \in I$. Replace $r$ by $r y$ and $y$ by $r y$, we obtain $[x, s] r y[x, p] r y[x, f(t)]=0$ for all $x, y \in I$ and $p, r, s \in I$. In particular, $[x, f(t)] r y[x, f(t)] r y[x, f(t)]=0$ for all $x, y \in I, r \in I$ and $t \in Z(R)$. It implies $(R y[x, f(Z(R))])^{3}=(0)$ for all $x, y \in I$. But $\mathrm{R}$ has no nonzero nilpotent ideal, so we have $R y[x, f(Z(R))]=(0)$ for all $x, y \in I$. Hence, we conclude that $I[x, f(Z(R))]=(0)$ for all $x \in I$.

Theorem 10. Let $R$ be a semiprime ring and I a nonzero left ideal of $R$. Suppose that $(F, f)$ is a multiplicative (generalized)derivation of $R$. If $F[x, y] \pm x y \in Z(R)$ holds for all $x, y \in I$, then $I[x, f(x)]=(0)$ or $I[x, f(Z(R))]=(0)$ for all $x \in I$.

Proof. We consider

$$
F[x, y] \pm x y \in Z(R) \text { for all } x, y \in I .
$$

If $Z(R)=(0)$ then it is easy to prove that $I[x, f(x)]=(0)$ for all $x \in I$.

If $Z(R) \neq(0)$ then there exist $0 \neq t \in Z(R)$. Replace $y$ by $y t$ in (45) to obtain $(F[x, y] \pm x y) t+[x, y] f(t) \in Z(R)$ for all $x, y \in I$. Using (45), we get $[x, y] f(t) \in Z(R)$ for all $x, y \in I$. On commuting with $r \in R$, we have $[[x, y] f(t), r]=0$ for all $x, y \in I$ and $r \in R$. It coincides with (32), hence Theorem 9. insure the conclusions. 
Theorem 11. Let $R$ be a semiprime ring and I a nonzero left ideal of $R$. Suppose that $(F, f)$ is a multiplicative (generalized)derivation of $R$. If $F(x \circ y) \pm x y \in Z(R)$ holds for all $x, y \in I$, then $I[x, f(x)]=(0)$ or $I[x, f(Z(R))]=(0)$ for all $x \in I$.

Proof. We consider

$$
F(x \circ y) \pm x y \in Z(R) \text { for all } x, y \in I .
$$

If $Z(R)=(0)$ then it is easy to prove that $I[x, f(x)]=(0)$ for all $x \in I$.

If $Z(R) \neq(0)$ then there exist $0 \neq t \in Z(R)$. Replace $y$ by $y t$ in (46) and we get $(F[x, y] \pm x y) t+(x \circ y) f(t) \in Z(R)$ for all $x, y \in I$. Using (46), we get $(x \circ y) f(t) \in Z(R)$ for all $x, y \in I$. On commuting with $r \in R$, we obtain $[(x \circ y) f(t), r]=0$ for all $x, y \in I$ and $r \in R$. It coincides with (40), hence Theorem 10. insure the conclusions.

Theorem 12. Let $R$ be a semiprime ring and I a nonzero left ideal of $R$. Suppose that $(F, f)$ is a multiplicative (generalized)derivation of $R$. If $F[x, y] \pm f(x) \circ y \in Z(R)$ holds for all $x, y \in I$, then $I[x, f(x)]=(0)$ or $I[x, f(Z(R))]$ for all $x \in I$.

Proof. We consider

$$
F[x, y] \pm f(x) \circ y \in Z(R) \text { for all } x, y \in I \text {. }
$$

If $Z(R)=(0)$ then we have

$$
F[x, y] \pm f(x) \circ y=0 \text { for all } x, y \in I .
$$

Substitute $y x$ for $y$ in (48) to get $(F[x, y] \pm f(x) \circ y) x+[x, y] f(x) \mp y[f(x), x]=0$ for all $x, y \in I$. By (48), it reduces to

$$
[x, y] f(x) \mp y[f(x), x]=0 \text { for all } x, y \in I .
$$

Replace $y$ by $f(x) y$ in (49), we get

$$
f(x)[x, y] f(x)+[x, f(x)] y f(x) \mp f(x) y[f(x), x]=0 \text { for all } x, y \in I .
$$

Left multiply (49) by $f(x)$ and subtract from (50), we obtain $[x, f(x)] y f(x)=0$ for all $x, y \in I$.. Since I is a left ideal in R, it implies that $y[x, f(x)] R y[x, f(x)]=(0)$ for all $x, y \in I$. Semiprimeness of R yields that $y[x, f(x)]=0$ for all $x, y \in I$. We conclude that $I[x, f(x)]=(0)$ for all $x \in I$.

If $Z(R) \neq(0)$ then there exist some $0 \neq t \in Z(R)$. Replace $y$ by $y t$ in $(47)$, we get $(F[x, y]+f(x) \circ y) t+[x, y] f(t) \in Z(R)$ for all $x, y \in I$. Using (47) to obtain $[x, y] f(t) \in Z(R)$ for all $x, y \in I$. That is $[[x, y] f(t), r]=0$ for all $x, y \in I$ and $r \in R$. It coincides with (32), hence Theorem 9. yields that $I[x, f(Z(R))]=(0)$ for all $x \in I$.

Theorem 13. Let $R$ be a semiprime ring and I a nonzero left ideal of $R$. Suppose that $(F, f)$ is a multiplicative (generalized)derivation of $R$. If $F(x \circ y) \pm[f(x), y] \in Z(R)$ holds for all $x, y \in I$, then $I[x, f(x)]=(0)$ or $I[x, f(Z(R))]=(0)$ for all $x \in I$.

Proof. We consider

$$
F(x \circ y) \pm[f(x), y] \in Z(R) \text { for all } x, y \in I \text {. }
$$

If $Z(R)=(0)$ then we have

$$
F(x \circ y) \pm[f(x), y]=0 \text { for all } x, y \in I .
$$

Replace $y$ by $y x$ in (52) and we obtain $F(x \circ y) x+(x \circ y) f(x) \pm[f(x), y] x \pm y[f(x), x]=0$ for all $x, y \in I$. Using (52), we left with

$$
(x \circ y) f(x) \pm y[f(x), x]=0 \text { for all } x, y \in I .
$$

Replace $y$ by $f(x) y$ in (53) and we get

$$
f(x)(x \circ y) f(x)+[x, f(x)] y f(x) \pm f(x) y[f(x), x]=0 \text { for all } x, y \in I .
$$

Left multiply (53) by $f(x)$ and subtract it from (54), we obtain $[x, f(x)] y f(x)=0$ for all $x, y \in I$. It implies that $[x, f(x)] y[x, f(x)]=(0)$ for all $x, y \in I$. Semiprimeness of R yields that $y[x, f(x)]=(0)$ for all $x, y \in I$. We conclude that $I[x, f(x)]=(0)$ for all $x \in I$.

If If $Z(R) \neq(0)$ then there exist some $0 \neq t \in Z(R)$. Replace $y$ by $y t$ in $(51)$, we get $(F(x \circ y)+[f(x), y]) t+(x \circ y) f(t) \in Z(R)$ for all $x, y \in I$. Using (51), we obtain $(x \circ y) f(t) \in Z(R)$ for all $x, y \in I$. That is $[(x \circ y) f(t), r]=0$ for all $x, y \in I$ and $r \in R$. It coincides with (40), hence Theorem 10. yields that $I[x, f(Z(R))]=(0)$ for all $x \in I$. 
Corollary 14. Let $R$ be a semi-prime ring. Suppose that $(F, f)$ is a multiplicative (generalized)-derivation of $R$. If any one of the following
i. $F[x, y] \pm(x \circ y) \in Z(R)$
ii. $F(x \circ y) \pm[x, y] \in Z(R)$
iii. $F[x, y] \pm x y \in Z(R)$
iv. $F(x \circ y) \pm x y \in Z(R)$
v. $F[x, y] \pm(f(x) \circ y) \in Z(R)$
vi. $F(x \circ y) \pm[f(x), y] \in Z(R)$

holds for all $x, y \in R$, then either $f$ is commuting map or $f(Z(R)) \subseteq Z(R)$.

\section{Examples}

In this section, we build a few examples to show that the condition of semiprimeness in our results is not superfluous.

Example 1. Consider

$$
R=\left\{\left(\begin{array}{ccc}
0 & a & b \\
0 & 0 & c \\
0 & 0 & 0
\end{array}\right): a, b, c \in S\right\}
$$

where $S$ is any arbitrary ring.

We define maps $F, f: R \rightarrow R$ by

$$
F\left(\begin{array}{ccc}
0 & a & b \\
0 & 0 & c \\
0 & 0 & 0
\end{array}\right)=\left(\begin{array}{ccc}
0 & 0 & a \\
0 & 0 & b c \\
0 & 0 & 0
\end{array}\right), f\left(\begin{array}{ccc}
0 & a & b \\
0 & 0 & c \\
0 & 0 & 0
\end{array}\right)=\left(\begin{array}{ccc}
0 & 0 & c^{2} \\
0 & 0 & 0 \\
0 & 0 & 0
\end{array}\right),
$$

it is verified that $F$ is a multiplicative (generalized)-derivations associated with the maps $f$ and it is easy to see that the identities $f(x) F(y) \pm[x, y] \in Z(R), f(x) F(y) \pm(x \circ y) \in Z(R)$ and $f(x) F(y) \pm y x \in Z(R)$ are satisfied for all $x, y \in R$. Here $\mathrm{R}$ is not a semiprime ring because

$$
\left(\begin{array}{lll}
0 & 1 & 1 \\
0 & 0 & 0 \\
0 & 0 & 0
\end{array}\right) R\left(\begin{array}{lll}
0 & 1 & 1 \\
0 & 0 & 0 \\
0 & 0 & 0
\end{array}\right)=(0)
$$

Note that $R$ is not commutative. Hence, the condition of semi-primeness in Corollary 5. can not be omitted.

Example 2. Consider $R=\left\{\left(\begin{array}{ll}a & b \\ 0 & c\end{array}\right): a, b, c \in \mathbb{Z}_{2}\right\}$ be a ring over integers modulo 2 and let $I=\left\{\left(\begin{array}{ll}a & b \\ 0 & 0\end{array}\right): a, b, c \in \mathbb{Z}_{2}\right\}$, be a left ideal in $\mathrm{R}$. We define maps $F, f: R \rightarrow R$ by

$$
F\left(\begin{array}{cc}
a & b \\
0 & c
\end{array}\right)=\left(\begin{array}{cc}
a & n b \\
0 & 0
\end{array}\right), f\left(\begin{array}{cc}
a & b \\
0 & c
\end{array}\right)=\left(\begin{array}{cc}
0 & (n-1) b \\
0 & 0
\end{array}\right),
$$

where $\mathrm{n}$ is any positive integer. Then it is verified that $F$ is a multiplicative (generalized)-derivations associated with the maps $f$ and it is easy to see that the identities $F(x y) \pm F(x) F(y) \in Z(R)$ are satisfied for all $x, y \in I$. Here $\mathrm{R}$ is not a semiprime ring, but observe that $I[f(x), x] \neq(0)$ for all $x \in I$. Hence, the condition of semiprimeness in Theorem 6 . is essential.

Example 3. Consider $R=\left\{\left(\begin{array}{lll}0 & a & b \\ 0 & 0 & c \\ 0 & 0 & 0\end{array}\right): a, b, c \in \mathbb{Z}\right\}$, where $\mathbb{Z}$ stands for the ring of integers. We define maps $F, f:$ $R \rightarrow R$ by 


$$
F\left(\begin{array}{ccc}
0 & a & b \\
0 & 0 & c \\
0 & 0 & 0
\end{array}\right)=\left(\begin{array}{ccc}
0 & 0 & b c \\
0 & 0 & 0 \\
0 & 0 & 0
\end{array}\right), f\left(\begin{array}{ccc}
0 & a & b \\
0 & 0 & c \\
0 & 0 & 0
\end{array}\right)=\left(\begin{array}{ccc}
0 & b & a^{2} \\
0 & 0 & 0 \\
0 & 0 & 0
\end{array}\right)
$$

Then it is verified that $F$ is a multiplicative (generalized)-derivations associated with the maps $f$ and it is easy to see that the identities $F[x, y] \pm(x \circ y) \in Z(R), F(x \circ y) \pm[x, y] \in Z(R), F[x, y] \pm x y \in Z(R), F(x \circ y) \pm x y \in Z(R), F[x, y] \pm(f(x) \circ y) \in Z(R)$ and $F(x \circ y) \pm[f(x), y] \in Z(R)$ are satisfied for all $x, y \in R$. Clearly, $\mathrm{R}$ is not a semiprime ring. Note that $f$ is neither commuting on $\mathrm{R}$ nor maps $Z(R)$ into $Z(R)$. Hence, the condition of semiprimeness in Corollary 14. can not be removed.

\section{References}

Ali, A., Dhara, B., Khan, S. \& Ali, F. (2015). Multiplicative (generalized)-derivations and left ideals in semiprime rings. Hecettape J. Math. Stat., 44(6), 1293-1306. http://dx.doi.org/10.15672/HJMS.2015449679

Ali, A., Kumar, D. \& Miyan, P. (2011). On generalized derivations and commutativity of prime and semiprime rings. Hecettepe J. Math. Stat., 40(3), 367-374.

Ali, S., Dhara, B., Dar N. A. \& Khan A.N. (2014). On Lie ideals with multiplicative (generalized)-derivations in prime and semiprime rings. Beitr. Algebra Geom., 56(1), 325-337. http://dx.doi.org/10.1007/s13366-013-0186-y

Ali, S., Dhara, B. \& Fošner, A. (2011). Some commutativity theorems concerning additive maps and derivations on semiprime rings. Contemp. Ring Theory: World Sci. Publ. Hackensack, NJ 2012, 135-143. http://dx.doi.org/10.1142 /9789814397681_0012

Andima, S. \& Pajoohesh, H. (2010). Commutativity of rings with derivations. Acta Math. Hung., 128(1-2), 1-14. http://dx.doi.org/10.1007/s10474-010-9092-z

Ashraf, M., Ali, A. \& Ali, S. (2007). Some commutativity theorems for rings with generalized derivations. Southeast Asian Bull. Math., 31, 415-421.

Ashraf, M. \& Rehman, N. (2001). On derivations and commutativity in prime rings. East-West J. Math., 3(1), 87-91.

Brešar, M. (1991). On the distance of composition of two derivations to the generalized derivation. Glasgow Math. J., 33, 89-93. http://dx.doi.org/10.1017/s0017089500008077

Daif, M. N. (1991). When is a multiplicative derivation additive? Internat. J. Math. Math. Sci., 14(3), 615-618. http://dx.doi.org/10.1155/s0161171291000844

Daif, M. N. \& Bell, H. E. (1992). Remarks on derivations on semiprime rings. Internat. J. Math. Math. Sci., 15(1), 205-206. http://dx.doi.org/10.1155/s0161171292000255

Daif, M. N. \& Tammam-El-Sayiad, M. S. (1997). Multiplicative generalized derivations which are additive. East-West J. Math., 9(1), 33-37.

Dhara, B. \& Ali, S. (2013). On multiplicative (generalized)-derivations in prime and semiprime rings. Aequations Math., 86(1-2), 65-79. http://dx.doi.org/10.1007/s00010-013-0205-y

Dhara, B., Kar, S. \& Das, D. (2014). A multiplicative (generalized)-( $\sigma, \sigma)$-derivations acting as (anti-) homomorphism in semiprime rings. Palestine J. Math., 3(2), 240-246.

Dhara, B. \& Pattanayak, A. (2011). Generalized derivations and left ideals in prime and semiprime rings. ISRN Algebra, Article id 750382, P.5. http://dx.doi.org/10.5402/2011/750382

Goldmann, H. \& Sěmrl, P. (1996). Multiplicative derivation on C(X). Monatsh. Math., 121(3), 189-197. http://dx.doi.org/1 $0.1007 / \mathrm{bf0} 1298949$

Havala, B. (1998). Generalized derivation in rings. Comm. Algebra, 26(4), 1147-1166. http://dx.doi.org/10.1080/00927879 808826190

Herstein, I. N. (1976). Rings with involution, The University of Chicago Press, Chicago.

Hongan, M. (1997). A note on semiprime rings with derivations. Internat J. Math. Math. Sci., 20, 413-415.

Khan, S. (2016). On semiprime rings with multiplicative (generalized)-derivations. Beitr. Algebra Geom., 57(1), $119-128$. http://dx.doi.org/10.1007/s13366-015-0241-y

Martindale III, W.S. (1969). When are multiplicative maps additive. Proc. Amer. Math. Soc., 21, 695-698. http://dx.doi. org/10.1090/s0002-9939-1969-0240129-7 


\section{Copyrights}

Copyright for this article is retained by the author(s), with first publication rights granted to the journal.

This is an open-access article distributed under the terms and conditions of the Creative Commons Attribution license (http://creativecommons.org/licenses/by/4.0/). 